\title{
The Effect of Bariatric Surgery Volume on General Surgery Outcomes for Morbidly Obese Patients
}

\author{
Katheryn Hope Wilkinson $\left(\mathbb{D},{ }^{1}\right.$ Ruizhe Wu, ${ }^{2}$ Aniko Szabo, ${ }^{2}$ Rana Higgins, ${ }^{1}$ Jon Gould, ${ }^{1}$ \\ and Tammy Kindel ${ }^{1}{ }^{1}$ \\ ${ }^{1}$ Department of Surgery, Medical College of Wisconsin, 8900 W Doyne Avenue, Milwaukee, WI 53226, USA
${ }^{2}$ Division of Biostatistics, Institute for Health and Equity, Medical College of Wisconsin, 8900 W Doyne Avenue, Milwaukee,
WI 53226, USA \\ Correspondence should be addressed to Tammy Kindel; tkindel@mcw.edu
}

Received 8 September 2021; Accepted 6 October 2021; Published 31 October 2021

Academic Editor: Francesco Saverio Papadia

Copyright (C) 2021 Katheryn Hope Wilkinson et al. This is an open access article distributed under the Creative Commons Attribution License, which permits unrestricted use, distribution, and reproduction in any medium, provided the original work is properly cited.

\begin{abstract}
Introduction. Bariatric surgery performed at high volume centers decreases length of stay, cost, and morbidity and mortality. The effect of a high volume of bariatric surgery procedures on outcomes may extend not just to bariatric surgery but to any general surgical procedure in morbidly obese patients. We hypothesized that patients with morbid obesity (body mass index $>40 \mathrm{~kg} / \mathrm{m}^{2}$ ) undergoing common, nonbariatric general surgery would have decreased morbidity and mortality at centers performing high volumes of bariatric surgery. Methods. The 2016 National Inpatient Sample (NIS) was used to identify the number of laparoscopic gastric bypass and sleeve gastrectomy performed at each hospital. Hospitals were classified as high volume bariatric hospitals $(\mathrm{HVBH}) \geq 10$ reported cases $(50$ actual)/year or low volume bariatric hospitals (LVBH) $<10$ reported cases $(50$ actual)/year, as NIS reports a $20 \%$ sample of actual cases. Patients with morbid obesity undergoing laparoscopic or open appendectomy, cholecystectomy, or ventral hernia repair were included for analysis. Propensity scores were developed based on available demographics, comorbidities, and hospital procedure volume. Postoperative complications during the index hospital admission, determined by ICD-10 code, were compared using inverse propensity weights. Differences were considered significant with a $p$ value of $<0.05$. Results. The total number of general surgery patient cases analyzed was 14,028 from 2,482 hospitals, representing 70,140 admissions. The cohort of patients undergoing operations treated at $\mathrm{HVBH}$ were younger $(p=0.03)$ with higher rates of COPD $(p=0.04)$. Patients at LVBH had higher rates of nicotine dependence $(p=0.0001)$ and obstructive sleep apnea $(p<0.001)$. On propensity-weighted analysis adjusting for preoperative comorbidities and hospital procedure volume, there were significantly higher rates of multiple postprocedure complications at LVBH, specifically, postprocedure respiratory failure for patients undergoing elective laparoscopic cholecystectomy, elective ventral hernia repair with mesh and appendectomy. Conclusion. Patients with morbid obesity may have an advantage in having general surgery procedures at HVBH. HVBH may have a volumeoutcomes relationship where the hospital and staff familiarity with the management principles required to minimize the postoperative risk associated with morbid obesity and improve patient outcomes.
\end{abstract}

\section{Introduction}

Patients with morbid obesity (body mass index, BMI $>40 \mathrm{~kg} /$ $\mathrm{m}^{2}$ ) have an increased risk of perioperative complications compared to normal weight individuals $[1,2]$. These increased risks include wound complications, renal complications, venous thromboembolic (VTE) events, and pulmonary complications that vary by procedure [1-6].
Any strategy to reduce the increased risk of perioperative complications in morbidly obese patients is important, including the known benefit of VTE chemoprophylaxis, preoperative diagnosis and treatment of obstructive sleep apnea, and preoperative glycemic control. These pathways are a prominent part of perioperative patient care in accredited and high volume bariatric centers. Bariatric surgery performed at high volume centers decreases the 
length of stay, cost, and morbidity and mortality $[7,8]$. The effect of a high volume of bariatric surgery procedures on outcomes may extend not just to bariatric surgery but to any general surgical procedure, as the hospital facility and staff are experienced in the surgical care of morbidly obese patients. We hypothesized that patients with morbid obesity undergoing common general surgery procedures would have decreased mortality and morbidity at centers performing high volumes of bariatric surgery.

\section{Methods}

2.1. Database. We used the 2016 National Inpatient Sample (NIS) to evaluate complication rates in patients with morbid obesity following traditionally, low-risk general surgery procedures. The NIS is a sample of $20 \%$ of discharges from all hospitals participating in the Healthcare Cost and Utilization Project. The NIS is unique in that patient information is linked to a unique hospital identifier.

2.2. Bariatric Surgery Volume. To estimate the number of bariatric surgeries performed by each hospital, the data was filtered for patients who underwent procedure codes of LRYGB (0D164ZA, 0D164ZB) and LSG (0DB64ZZ) with a diagnosis of morbid obesity (E66.01, E66.2). Patients were excluded if they had gastrointestinal neoplasm (C15-C26), inflammatory bowel disease (K50-51), or noninfectious colitis (K52). The number of cases for each hospital was attached to the NIS hospital number. Current metabolic and bariatric surgery quality and improvement program (MBSAQIP) center accreditation as a comprehensive center requires a program to perform $\geq 50$ bariatric stapling surgeries per year. As the NIS data contains information for $20 \%$ of hospital discharges, we used a bariatric caseload of 10 or more as the threshold to define hospitals performing high volumes of bariatric surgery $(\mathrm{HVBH})$ compared to low volume bariatric hospitals (LVBH).

2.3. Study Cohorts. We included patients with a diagnosis of morbid obesity $\left(\mathrm{BMI}>40 \mathrm{~kg} / \mathrm{m}^{2}\right)$ who underwent nonelective or elective laparoscopic cholecystectomy (0FB40ZZ, 0FB44ZZ, 0FT44ZZ, 0FT40ZZ) and laparoscopic or open ventral hernia repair with (0WUF0JZ, 0WUF4JZ, 0WUF0KZ, 0WUF4KZ) or without mesh (0WQF0ZZ, OWQF4ZZ). We included patients who underwent nonelective laparoscopic or open appendectomy (0DBJ0ZZ, 0DBJ4ZZ, 0DTJ0ZZ, 0DTJ4ZZ), and we excluded all appendectomies that were scheduled as elective due to inadequate procedure numbers for analysis.

2.4. Outcomes. Our primary outcomes of interest were postoperative complications, mortality, and length of stay. Complications were identified by ICD-10 codes (see Supplemental Table A) like methods previously described [9]. If a patient experienced one or more of the 24 complications, they were considered to have "any complication."
2.5. Statistics. Descriptive statistics of preoperative demographics and comorbidities were compared with surveyweighted chi-squared tests adjusting for within-hospital clustering. Statistical analysis was performed using SAS version 9.4. Statistical significance was defined as $p<0.05$.

2.6. Propensity Analysis. Propensity scores for the probability of having a procedure in a $\mathrm{HVBH}$ versus $\mathrm{LVBH}$ hospital were computed separately for each procedure and overall for the entire study population using the following predictors: age, median household income national quartile for patient ZIP Code, gender, renal failure, hypertension, chronic obstructive pulmonary disease, diabetes mellitus type 2, anemia, gastroesophageal reflux disease, chronic peptic ulcer disease, heart failure, hypothyroidism, history of VTE, history of pulmonary embolism (PE), nicotine dependence, obstructive sleep apnea, and the volume of the relevant procedure among all patients (i.e., the overall appendectomy volume when modeling the appendectomy population, etc.). Average treatment effect (ATE) weights were computed based on the propensity score, and weighted analysis was performed to estimate the effect of high bariatric volume versus low bariatric volume on the outcomes. Positive values imply higher rates in high bariatric volume hospitals. The propensity score weighted analysis was conducted with SAS version 9.4 PROC CAUSALTRT.

\section{Results}

3.1. All Procedures. The overall study cohort included 3,867 cases at $\mathrm{HVBH}$ and 10,161 cases at LVBH. The $\mathrm{HVBH}$ patients were younger (50.5 years vs. 51.2 years $p=0.03$ ), with a shorter length of stay (4.85 days vs. 5.39 days, $p<0.0001)$. Patients at HVBH also were more likely to be female and carry a preoperative diagnosis of hypertension, GERD, history of VTE or PE, nicotine dependence, and obstructive sleep apnea; see Table 1. Patients at LVBH were more likely to have preoperative renal failure. In unadjusted comparisons, postoperative rates of bowel obstruction $(2.8 \%$ vs. $2.0 \%, p=0.01)$, pulmonary failure $(2.9 \%$ vs. $1.6 \%$ $p<0.0001)$, and any complication $(10.3 \%$ v. $7.76 \%,<0.0001)$ were higher at $\mathrm{LVBH}$ compared to $\mathrm{HVBH}$. On propensityweighted analysis, controlling for the entire set of preoperative comorbidities and the hospital volume differences, patients at $\mathrm{LVBH}$ were still more likely to have bowel obstructions $(p=0.012)$, pulmonary failure $(<0.0001)$, postoperative infection (0.005), wound disruption $(p=0.03)$, or any postoperative complication $(p<0.0001)$.

3.2. Elective Laparoscopic Cholecystectomy. In the study cohort, 942 patients underwent elective laparoscopic cholecystectomy at $\mathrm{HVBH}$ (weighted $n=4,710$ ) and 823 patients underwent elective laparoscopic cholecystectomy at $\mathrm{LVBH}$ (weighted $n=4,115)$. Patients at HVBH were younger (47.8 years vs. 51.0 years, $p<0.0001)$ and had shorter lengths of stay (3.13 days vs. 4.09 days, $p<0.0001$ ); see Table 2. Patients had similar rates of many comorbidities; however, more patients at LVBH had COPD (7.86\% vs. $5.22 \%, p=0.03)$ 
TABLE 1: Preoperative comorbidities and postoperative complications of patients with morbid obesity undergoing cholecystectomy, appendectomy, or ventral hernia repair.

\begin{tabular}{|c|c|c|c|c|c|}
\hline Preoperative variables & $\begin{array}{c}\text { HVBH } n=3,867 \\
\text { Weighted } \\
n=19,335\end{array}$ & $\begin{array}{c}\text { LVBH } n=10,161 \\
\text { Weighted } \\
n=50,805\end{array}$ & $\begin{array}{c}p \text { value } \\
\text { unadjusted }\end{array}$ & & \\
\hline Age (years, mean $\pm S E)$ & $50.5 \pm 0.3$ & $51.2 \pm 0.2$ & 0.03 & & \\
\hline Length of stay (days, mean $\pm S E$ ) & $4.9 \pm 0.1$ & $5.4 \pm 0.1$ & $<0.0001$ & & \\
\hline $\begin{array}{l}\text { Median household income quartile for zip } \\
\text { code }\end{array}$ & $2.32 \pm 0.04$ & $2.25 \pm 0.02$ & 0.0005 & & \\
\hline Gender, female $(\% \pm S E)$ & $72.7 \pm 0.8$ & $69.4 \pm 0.5$ & 0.0003 & & \\
\hline Renal failure $(\% \pm S E)$ & $1.1 \pm 0.2$ & $1.5 \pm 0.1$ & 0.08 & & \\
\hline Hypertension $(\% \pm S E)$ & $52.4 \pm 0.9$ & $49.3 \pm 0.5$ & 0.002 & & \\
\hline Chronic obstructive pulmonary sis. $(\% \pm S E)$ & $8.9 \pm 0.5$ & $10.3 \pm 0.3$ & 0.03 & & \\
\hline Diabetes mellitus type $2(\% \pm S E)$ & $10.2 \pm 0.6$ & $9.8 \pm 0.3$ & 0.51 & & \\
\hline Anemia $(\% \pm S E)$ & $0.78 \pm 0.18$ & $0.53 \pm 0.07$ & 0.15 & & \\
\hline Gastroesophageal reflux disease $(\% \pm S E)$ & $33.2 \pm 1.0$ & $24.9 \pm 0.5$ & $<0.0001$ & & \\
\hline Chronic peptic ulcer disease $(\% \pm S E)$ & $0.72 \pm 0.15$ & $0.82 \pm 0.09$ & 0.61 & & \\
\hline Heart failure $(\% \pm S E)$ & $7.5 \pm 0.5$ & $8.4 \pm 0.3$ & 0.10 & & \\
\hline Hypothyroidism $(\% \pm S E)$ & $12.7 \pm 0.5$ & $12.7 \pm 0.3$ & 0.97 & & \\
\hline $\begin{array}{l}\text { History of venous thromboembolism (\% } \pm \\
\text { SE) }\end{array}$ & $4.1 \pm 0.4$ & $3.1 \pm 0.2$ & 0.005 & & \\
\hline History of pulmonary embolism $(\% \pm S E)$ & $3.4 \pm 0.3$ & $2.1 \pm 0.1$ & $<0.0001$ & & \\
\hline Nicotine dependence $(\% \pm S E)$ & $20.1 \pm 0.9$ & $16.6 \pm 0.4$ & 0.0001 & & \\
\hline Obstructive sleep apnea $(\% \pm S E)$ & $35.2 \pm 1.3$ & $24.8 \pm 0.5$ & $<0.0001$ & & \\
\hline Postprocedure complications & $\mathrm{HVBH}$ & LVBH & $\begin{array}{c}p \text { value } \\
\text { unadjusted }\end{array}$ & $\operatorname{ATE}(95 \% \mathrm{CI})$ & $\begin{array}{l}p \text { value } \\
\text { adjusted }\end{array}$ \\
\hline Cerebrovascular infarct $(\% \pm S E)$ & $0.03 \pm 0.03$ & $0.03 \pm 0.02$ & 0.91 & - & - \\
\hline Shock $(\% \pm S E)$ & $0.39 \pm 0.12$ & $0.29 \pm 0.05$ & 0.39 & - & - \\
\hline Hemorrhage or hematoma $(\% \pm S E)$ & $0.18 \pm 0.07$ & $0.18 \pm 0.04$ & 0.96 & - & - \\
\hline Bowel obstruction $(\% \pm S E)$ & $1.99 \pm 0.26$ & $2.82 \pm 0.18$ & 0.01 & $-0.76(-1.36 / 0.16)$ & 0.01 \\
\hline Enterotomy $(\% \pm S E)$ & $0.57 \pm 0.13$ & $0.67 \pm 0.08$ & 0.53 & - & - \\
\hline GI bleed $(\% \pm S E)$ & $0.41 \pm 0.10$ & $0.53 \pm 0.07$ & 0.36 & $-0.19(-0.44 / 0.06)$ & 0.13 \\
\hline Pulmonary insufficiency $(\% \pm S E)$ & $0.31 \pm 0.10$ & $0.2 \pm 0.05$ & 0.25 & - & - \\
\hline Pulmonary failure $(\% \pm S E)$ & $1.55 \pm 0.21$ & $2.9 \pm 0.18$ & $<0.0001$ & $\begin{array}{c}-1.31(-1.93 / \\
-0.69)\end{array}$ & $<0.0001$ \\
\hline Pneumonia $(\% \pm S E)$ & $0.41 \pm 0.11$ & $0.5 \pm 0.07$ & 0.51 & - & - \\
\hline Genitourinary complication $(\% \pm S E)$ & $0.16 \pm 0.06$ & $0.14 \pm 0.04$ & 0.81 & - & - \\
\hline Postoperative infection $(\% \pm S E)$ & $1.14 \pm 0.19$ & $1.46 \pm 0.11$ & 0.18 & $\begin{array}{c}-0.59(-1.01 / \\
-0.18)\end{array}$ & 0.005 \\
\hline Wound complication $(\% \pm S E)$ & $0.16 \pm 0.06$ & $0.12 \pm 0.03$ & 0.58 & $\begin{array}{l}-0.42(-0.80 / \\
-0.03)\end{array}$ & 0.03 \\
\hline Venous thromboembolism $(\% \pm S E)$ & $0.31 \pm 0.09$ & $0.45 \pm 0.07$ & 0.24 & - & - \\
\hline Pulmonary embolism $(\% \pm S E)$ & $0.65 \pm 0.14$ & $0.54 \pm 0.07$ & 0.49 & - & - \\
\hline "Other" complications $(\% \pm S E)$ & $0.18 \pm 0.07$ & $0.27 \pm 0.05$ & 0.36 & - & - \\
\hline Death $(\% \pm S E)$ & $0.59 \pm 0.12$ & $0.8 \pm 0.09$ & 0.19 & $-0.10(-0.47 / 0.27)$ & 0.60 \\
\hline Patient had any complication $(\% \pm S E)$ & $7.8 \pm 0.5$ & $10.3 \pm 0.3$ & $<0.0001$ & $\begin{array}{c}-3.32(-4.43 / \\
-2.21) \\
\end{array}$ & $<0.0001$ \\
\hline
\end{tabular}

HVBH: high volume bariatric hospital; LVBH: low volume bariatric hospital; ATE: average treatment effect.

and heart failure $(6.79 \%$ vs. $4.13 \%, p=0.01)$ and more patients at HBVH had anemia $(1.94 \%$ vs. $0.75 \%, p=0.05)$ and OSA $(42.9 \%$ vs. $33.7 \%, p=0.01)$. HVBH had higher levels of cholecystectomy volume (62.2/hospital vs. 40.4/ hospital, $p<0.001)$. LVBH had higher rates of postoperative pulmonary failure $(4.14 \%$ vs. $0.85 \%, p<0.0001)$ and any complication $(9.45 \%$ vs. $5.95 \%, p=0.01)$. On propensityweighted analysis, patients at LVBH still had higher rates of pulmonary failure $(p<0.0001)$ any complication $(p=0.04)$, and a significantly increased risk of postoperative mortality $(p=0.05)$.
3.3. Nonelective Laparoscopic Cholecystectomy. In the study cohort, 1,047 patients underwent nonelective laparoscopic cholecystectomy at HVBH (weighted $n=5,235$ ) and 4,399 patients underwent nonelective laparoscopic cholecystectomy at LVBH (weighted $n=21,995$ ); see Table 3 . Patients at LVBH had shorter lengths of stay (4.87 days vs. 5.25 days, $p=0.05$ ). Patients had similar rates of many comorbidities; however, more patients at HVBH had preoperative GERD and OSA ( $27.7 \%$ vs. $23.1 \%, p=0.003$, and $23.4 \%$ vs. $19.5 \%$, $p=0.009$, respectively). HVBH had higher levels of nonelective cholecystectomy volume (54.9/hospital vs. 39.0/ 
TABle 2: Preoperative comorbidities and postoperative complications of patients with morbid obesity undergoing elective laparoscopic cholecystectomy.

\begin{tabular}{|c|c|c|c|c|c|}
\hline Preoperative variables & $\begin{array}{c}\text { HVBH } n=942 \\
\text { Weighted } \\
n=4710\end{array}$ & $\begin{array}{c}\text { LVBH } n=823 \\
\text { Weighted } \\
n=4115\end{array}$ & $\begin{array}{c}p \text { value } \\
\text { unadjusted }\end{array}$ & & \\
\hline Age (years, mean $\pm S E$ ) & $47.9 \pm 0.6$ & $51.0 \pm 0.5$ & $<0.0001$ & & \\
\hline Length of stay (days, mean $\pm S E$ ) & $3.3 \pm .02$ & $4.1 \pm 0.2$ & $<0.0001$ & & \\
\hline $\begin{array}{l}\text { Median household income quartile for zip } \\
\text { code }\end{array}$ & $2.5 \pm 0.05$ & $2.4 \pm 0.04$ & 0.05 & & \\
\hline Gender, female $(\% \pm S E)$ & $75.5 \pm 1.5$ & $71.5 \pm 1.4$ & 0.06 & & \\
\hline Renal failure $(\% \pm S E)$ & $0.73 \pm 0.30$ & $1.17 \pm 0.33$ & 0.34 & & \\
\hline Hypertension $(\% \pm S E)$ & $53.3 \pm 1.6$ & $52.4 \pm 1.5$ & 0.68 & & \\
\hline Chronic obstructive pulmonary dis. (\%土 SE) & $5.2 \pm 0.8$ & $7.9 \pm 0.9$ & 0.03 & & \\
\hline Diabetes mellitus type $2(\% \pm S E)$ & $9.5 \pm 1.0$ & $11.7 \pm 1.0$ & 0.12 & & \\
\hline Anemia $(\% \pm S E)$ & $1.94 \pm 0.71$ & $0.74 \pm 0.27$ & 0.05 & & \\
\hline Gastroesophageal reflux disease (\% SE) & $39.5 \pm 2.1$ & $36.9 \pm 1.9$ & 0.38 & & \\
\hline Chronic peptic ulcer disease (\% $(S E)$ & $0.49 \pm 0.30$ & $0.74 \pm 0.26$ & 0.54 & & \\
\hline Heart failure (\% $\%$ SE) & $4.1 \pm 0.7$ & $6.8 \pm 0.8$ & 0.01 & & \\
\hline Hypothyroidism (\% $\%$ SE) & $11.5 \pm 1.0$ & $12.5 \pm 1.0$ & 0.49 & & \\
\hline History of venous thromboembolism $(\% \pm S E)$ & $3.7 \pm 0.8$ & $2.6 \pm 0.5$ & 0.19 & & \\
\hline History of pulmonary embolism (\% $\mathrm{SE}$ ) & $2.3 \pm 0.5$ & $1.6 \pm 0.4$ & 0.25 & & \\
\hline Nicotine dependence $(\% \pm S E)$ & $21.4 \pm 2.1$ & $19.0 \pm 1.3$ & 0.30 & & \\
\hline Obstructive sleep apnea (\% SE) & $42.9 \pm 3.6$ & $33.7 \pm 1.6$ & 0.01 & & \\
\hline Cholecystectomy volume (cases/hospital $\pm S E$ ) & $62.2 \pm 8.1$ & $40.4 \pm 1.2$ & $<0.00$ & & \\
\hline Postprocedure complications & HVBH & LVBH & $\begin{array}{c}p \text { value } \\
\text { unadjusted }\end{array}$ & ATE $(95 \% \mathrm{CI})$ & $\begin{array}{c}p \text { value } \\
\text { adjusted }\end{array}$ \\
\hline Shock $(\% \pm S E)$ & $0.24 \pm 0.17$ & $0.42 \pm 0.21$ & 0.52 & - & - \\
\hline Hemorrhage or hematoma $(\% \pm S E)$ & $0.49 \pm 0.22$ & $0.42 \pm 0.21$ & 0.84 & - & - \\
\hline Bowel obstruction (\% $(S E)$ & $1.22 \pm 0.39$ & $1.91 \pm 0.45$ & 0.25 & $-0.50(-1.57 / 0.58)$ & 0.36 \\
\hline Enterotomy $(\% \pm S E)$ & $0.73 \pm 0.30$ & $0.64 \pm 0.24$ & 0.81 & $0.37(-0.35 / 1.10)$ & 0.32 \\
\hline GI bleed (\%土SE) & $0.49 \pm 0.25$ & $0.85 \pm 0.28$ & 0.34 & $-0.33(-1.10 / 0.43)$ & 0.39 \\
\hline Pulmonary insufficiency (\%土SE) & $0.12 \pm 0.12$ & $0.42 \pm 0.18$ & 0.22 & - & - \\
\hline 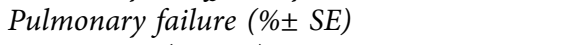 & $0.85 \pm 0.30$ & $4.14 \pm 0.67$ & $<0.0001$ & $-3.27(-4.8 /-1.73)$ & $<0.0001$ \\
\hline Pneumonia (\% $\mathrm{SE}$ ) & $0.61 \pm 0.28$ & $0.42 \pm 0.18$ & 0.57 & - & - \\
\hline Genitourinary complication (\% $\%$ SE) & $0.24 \pm 0.17$ & $0.21 \pm 0.15$ & 0.89 & - & - \\
\hline Postoperative infection (\% $\%$ SE) & $0.85 \pm 0.31$ & $0.85 \pm 0.30$ & 1.00 & $0.18(-0.90 / 1.26)$ & 0.74 \\
\hline Wound complication (\% $\%$ SE) & $0.12 \pm 0.12$ & $0.11 \pm 0.11$ & 0.92 & - & - \\
\hline Venous thromboembolism (\% $\%$ SE) & $0.12 \pm 0.12$ & $0.42 \pm 0.21$ & 0.23 & - & - \\
\hline Pulmonary embolism $(\% \pm S E)$ & $0.36 \pm 0.21$ & $0.32 \pm 0.18$ & 0.87 & - & - \\
\hline "Other" complications $(\% \pm S E)$ & $0.12 \pm 0.12$ & $0.11 \pm 0.11$ & 0.92 & - & - \\
\hline death $(\% \pm S E)$ & $0.24 \pm 0.17$ & $0.96 \pm 0.32$ & 0.06 & $-0.65(-1.30 / 0.01)$ & 0.05 \\
\hline Patient had any complication $(\% \pm S E)$ & $6.0 \pm 0.9$ & $9.5 \pm 1.0$ & 0.01 & $\begin{array}{c}-2.67(-5.25 / \\
-0.09)\end{array}$ & 0.04 \\
\hline
\end{tabular}

HVBH: high volume bariatric hospital; LVBH: low volume bariatric hospital; ATE: average treatment effect.

hospital, $p<0.001)$. After propensity weighting, LVBH had higher rates of postprocedure hemorrhage $(p=0.03)$ and any complication $(p=0.05)$.

3.4. Elective Ventral Hernia Repair. For patients undergoing elective ventral hernia repair, there were 514 patients at HVBH (weighted $n=2,570$ ) and 1,130 patients at LVBH (weighted $n=5,650$ ). As shown in Table 4, patients at HVBH had significantly higher rates of preoperative GERD, peptic ulcer disease, history of pulmonary embolism, nicotine dependence, and OSA. HVBH had higher rates of ventral hernia repair volume with an average of 22 cases/hospital versus 13 cases/hospital $(p<0.0001)$. LVBH had higher rates of postoperative pulmonary failure $(4.69 \%$ vs. $1.56 \%, p=0.002)$ and any complication $(16.4 \%$ vs.
$12.3 \%, p=0.03)$. After propensity weighting, patients at $\mathrm{LVBH}$ had still had higher rates of pulmonary failure $(p<0.0001)$ as well as having an increased risk of having any indiviudal complication $(p=0.01)$.

3.5. Nonelective Ventral Hernia Repair. For patients undergoing nonelective ventral hernia repair, there were 314 patients at $\mathrm{HVBH}$ (weighted $n=1,570$ ) and 1,143 patients at LVBH (weighted $n=5,715$ ). As shown in Table 5 , patients at HVBH had higher rates of history of pulmonary embolism, nicotine dependence, and OSA. HVBH had higher ventral hernia procedure volume with an average of 20.9 cases/ hospital versus 10.8 cases/hospital $(p<0.0001)$. After propensity weighting, patients at LVBH had higher rates of postoperative death $(p=0.03)$. 
TABLE 3: Preoperative comorbidities and postoperative complications of patients with morbid obesity undergoing nonelective laparoscopic cholecystectomy.

\begin{tabular}{|c|c|c|c|c|c|}
\hline Preoperative variables & $\begin{array}{c}\text { HVBH } n=1047 \\
\text { Weighted } \\
n=5235\end{array}$ & $\begin{array}{c}\text { LVBH } n=4399 \\
\text { Weighted } \\
n=21995\end{array}$ & $\begin{array}{c}p \text { value } \\
\text { unadjusted }\end{array}$ & & \\
\hline Age (years, mean $\pm S E)$ & $48.1 \pm 0.6$ & $48.5 \pm 0.3$ & 0.47 & & \\
\hline Length of stay (days, mean $\pm S E$ ) & $5.3 \pm 0.2$ & $4.9 \pm 0.1$ & 0.05 & & \\
\hline $\begin{array}{l}\text { Median household income quartile for zip } \\
\text { code }\end{array}$ & $2.2 \pm 0.1$ & $2.2 \pm 0.0$ & 0.49 & & \\
\hline Gender, female (\% $\%$ SE) & $27.4 \pm 1.4$ & $30.0 \pm 0.7$ & 0.10 & & \\
\hline Renal failure (\%土SE) & $1.8 \pm 0.4$ & $1.6 \pm 0.2$ & 0.58 & & \\
\hline Hypertension (\% $\%$ SE) & $45.5 \pm 1.6$ & $44.6 \pm 0.8$ & 0.65 & & \\
\hline $\begin{array}{l}\text { Chronic obstructive pulmonary dis. (\% } \\
\text { SE) }\end{array}$ & $8.2 \pm 0.9$ & $9.0 \pm 0.4$ & 0.48 & & \\
\hline Diabetes mellitus type $2(\% \pm S E)$ & $10.4 \pm 0.9$ & $8.9 \pm 0.4$ & 0.12 & & \\
\hline Anemia $(\% \pm S E)$ & $0.67 \pm 0.25$ & $0.36 \pm 0.09$ & 0.17 & & \\
\hline Gastroesophageal reflux disease (\% $\%$ SE) & $27.7 \pm 1.5$ & $23.1 \pm 0.7$ & 0.003 & & \\
\hline Chronic peptic ulcer disease (\% $(S E)$ & $0.76 \pm 0.27$ & $1.18 \pm 0.16$ & 0.24 & & \\
\hline Heart failure (\% $\%$ SE) & $10.0 \pm 1.0$ & $8.2 \pm 0.4$ & 0.08 & & \\
\hline Hypothyroidism (\% $\%$ SE) & $10.7 \pm 1.0$ & $11.6 \pm 0.5$ & 0.43 & & \\
\hline $\begin{array}{l}\text { History of venous thromboembolism (\% } \\
\text { SE) }\end{array}$ & $3.2 \pm 0.6$ & $2.7 \pm 0.2$ & 0.42 & & \\
\hline History of pulmonary embolism (\% $\%$ SE) & $2.1 \pm 0.4$ & $1.7 \pm 0.2$ & 0.36 & & \\
\hline Nicotine dependence $(\% \pm S E)$ & $17.1 \pm 1.2$ & $15.7 \pm 0.6$ & 0.31 & & \\
\hline Obstructive sleep apnea (\% $\%$ SE) & $23.4 \pm 1.4$ & $19.5 \pm 0.6$ & 0.009 & & \\
\hline $\begin{array}{l}\text { Cholecystectomy volume (cases/hospital } \pm \\
\text { SE) }\end{array}$ & $54.9 \pm 2.5$ & $39.0 \pm 0.9$ & $<0.0001$ & & \\
\hline Postprocedure complications & $\mathrm{HVBH}$ & LVBH & $\begin{array}{c}p \text { value } \\
\text { unadjusted }\end{array}$ & $\operatorname{ATE}(95 \% \mathrm{CI})$ & $\begin{array}{l}p \text { value } \\
\text { adjusted }\end{array}$ \\
\hline Shock $(\% \pm S E)$ & $0.19 \pm 0.13$ & $0.11 \pm 0.05$ & 0.53 & - & - \\
\hline Hemorrhage or hematoma $(\% \pm S E)$ & $0.10 \pm 0.10$ & $0.27 \pm 0.08$ & 0.29 & $\begin{array}{c}-0.20(-0.39 / \\
-0.02)\end{array}$ & 0.03 \\
\hline Cardiac arrest $(\% \pm S E)$ & $0.19 \pm 0.13$ & $0.05 \pm 0.03$ & 0.12 & $\begin{array}{c}-0.49(-1.01 / \\
0.03)\end{array}$ & 0.07 \\
\hline Bowel obstruction (\% $\%$ SE) & $0.86 \pm 0.28$ & $1.14 \pm 0.16$ & 0.43 & $\begin{array}{c}-0.19(-0.55 / \\
0.17)\end{array}$ & 0.31 \\
\hline Enterotomy $(\% \pm S E)$ & $0.38 \pm 0.19$ & $0.48 \pm 0.10$ & 0.68 & $\begin{array}{c}-0.17(-0.53 / \\
0.19)\end{array}$ & 0.36 \\
\hline GI bleed (\% $\%$ SE) & $0.19 \pm 0.14$ & $0.39 \pm 0.09$ & 0.33 & - & - \\
\hline Pulmonary failure $(\% \pm S E)$ & $1.2 \pm 0.3$ & $1.6 \pm 0.2$ & 0.33 & $\begin{array}{c}-0.43(-1.23 / \\
0.37)\end{array}$ & 0.29 \\
\hline Pneumonia (\% \pm SE) & $0.19 \pm 0.13$ & $0.25 \pm 0.07$ & 0.72 & $\begin{array}{c}-0.15(-0.39 / \\
0.08)\end{array}$ & 0.20 \\
\hline Genitourinary complication (\% $\$ S E$ ) & $0.19 \pm 0.13$ & $0.09 \pm 0.04$ & 0.38 & - & - \\
\hline Postoperative infection (\%土SE) & $0.29 \pm 0.16$ & $0.43 \pm 0.10$ & 0.50 & $\begin{array}{l}-0.24(-0.55 / \\
0.08)\end{array}$ & 0.14 \\
\hline Wound disruption $(\% \pm S E)$ & $0.29 \pm 0.16$ & $0.27 \pm 0.08$ & 0.94 & $\begin{array}{c}-0.17(-0.41 / \\
0.06)\end{array}$ & 0.15 \\
\hline Venous thromboembolism (\% $\%$ SE) & $0.10 \pm 0.10$ & $0.25 \pm 0.07$ & 0.34 & $\begin{array}{c}-0.17(-0.41 / \\
0.07)\end{array}$ & 0.17 \\
\hline Pulmonary embolism $(\% \pm S E)$ & $0.29 \pm 0.16$ & $0.30 \pm 0.08$ & 0.96 & $0.09(-0.38 / 0.56)$ & 0.70 \\
\hline "Other" complications $(\% \pm S E)$ & $0.10 \pm 0.10$ & $0.16 \pm 0.06$ & 0.63 & - & - \\
\hline Death $(\% \pm S E)$ & $0.67 \pm 0.25$ & $0.41 \pm 0.10$ & 0.26 & $0.22(-0.27 / 0.71)$ & 0.39 \\
\hline Patient had any complication $(\% \pm S E)$ & $3.82 \pm 0.58$ & $5.11 \pm 0.34$ & 0.08 & $\begin{array}{c}-1.88(-3.17 / \\
-0.58)\end{array}$ & 0.005 \\
\hline
\end{tabular}

HVBH: high volume bariatric hospital; LVBH: low volume bariatric hospital; ATE: average treatment effect.

3.6. Nonelective Appendectomy. The patients that underwent appendectomy (nonelective cases only) included 205 patients at $\mathrm{HVBH}$ (weighted $n=1,025$ ) and 870 patients at LVBH (weighted $n=4,350$ ). Patients at LVBH had shorter length of stay (3.88 days vs. 4.7 days, $p=0.02$ ); see Table 6 . There were no statistically significant differences in any preoperative comorbidity. There were more appendectomy cases done per hospital at $\mathrm{HVBH}(23.1 /$ hospital vs. 17.0/hospital, $p<0.0001)$. $\mathrm{LVBH}$ had higher rates of postoperative pulmonary 
TABLE 4: Preoperative comorbidities and postoperative complications of patients with morbid obesity undergoing elective ventral hernia repair.

\begin{tabular}{|c|c|c|c|c|c|}
\hline Preoperative variables & $\begin{array}{c}\text { HVBH } n=514 \\
\text { Weighted } \\
n=2570\end{array}$ & $\begin{array}{c}\text { LVBH } n=1130 \\
\text { Weighted } \\
n=5650\end{array}$ & $\begin{array}{c}p \text { value } \\
\text { unadjusted }\end{array}$ & & \\
\hline Age (years, mean $\pm S E$ ) & $54.8 \pm 0.5$ & $55.89 \pm 0.4$ & 0.08 & & \\
\hline Length of stay (days, mean $\pm S E$ ) & $5.1 \pm 0.3$ & $5.8 \pm 0.3$ & 0.17 & & \\
\hline $\begin{array}{l}\text { Median household income quartile for zip } \\
\text { code }\end{array}$ & $2.3 \pm 0.05$ & $2.3 \pm 0.03$ & 0.80 & & \\
\hline Gender, female $(\% \pm S E)$ & $72.2 \pm 2.2$ & $71.7 \pm 1.4$ & 0.87 & & \\
\hline Renal failure (\%土SE) & $0.39 \pm 0.28$ & $1.06 \pm 0.30$ & 0.17 & & \\
\hline Hypertension (\%土SE) & $57.4 \pm 2.1$ & $55.3 \pm 1.5$ & 0.42 & & \\
\hline $\begin{array}{l}\text { Chronic obstructive pulmonary dis. (\% } \$ \\
\text { SE) }\end{array}$ & $13.0 \pm 1.4$ & $13.5 \pm 1.0$ & 0.77 & & \\
\hline Diabetes mellitus type $2(\% \pm S E)$ & $10.9 \pm 1.4$ & $11.2 \pm 1.0$ & 0.88 & & \\
\hline Anemia $(\% \pm S E)$ & $0.58 \pm 0.34$ & $0.97 \pm 0.28$ & 0.42 & & \\
\hline Gastroesophageal reflux disease (\% $\%$ SE) & $35.2 \pm 2.3$ & $29.6 \pm 1.4$ & 0.03 & & \\
\hline Chronic peptic ulcer disease (\% $(S E)$ & $1.56 \pm 0.59$ & $0.44 \pm 0.20$ & 0.02 & & \\
\hline Heart failure $(\% \pm S E)$ & $5.8 \pm 1.0$ & $6.2 \pm 0.7$ & 0.78 & & \\
\hline 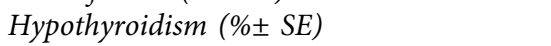 & $15.0 \pm 1.5$ & $14.7 \pm 1.0$ & 0.87 & & \\
\hline $\begin{array}{l}\text { History of venous thromboembolism (\% } \\
\text { SE) }\end{array}$ & $6.4 \pm 1.1$ & $4.3 \pm 0.6$ & 0.07 & & \\
\hline History of pulmonary embolism (\% $\%$ SE) & $6.4 \pm 1.0$ & $2.7 \pm 0.5$ & 0.0003 & & \\
\hline Nicotine dependence $(\% \pm S E)$ & $25.7 \pm 2.0$ & $18.9 \pm 1.1$ & 0.002 & & \\
\hline Obstructive sleep apnea (\% $\%$ SE) & $38.5 \pm 2.2$ & $30.1 \pm 1.3$ & 0.001 & & \\
\hline Ventral hernia volume (cases/hospital $\pm S E$ ) & $22.4 \pm 2.2$ & $13.2 \pm 0.6$ & $<0.0001$ & & \\
\hline Postprocedure complications & $\mathrm{HVBH}$ & LVBH & $\begin{array}{c}p \text { value } \\
\text { unadjusted }\end{array}$ & $\operatorname{ATE}(95 \% \mathrm{CI})$ & $\begin{array}{l}p \text { value } \\
\text { adjusted }\end{array}$ \\
\hline Shock $(\% \pm S E)$ & $0.97 \pm 0.63$ & $0.27 \pm 0.15$ & 0.11 & - & - \\
\hline Bowel obstruction (\% $\%$ SE) & $3.3 \pm 0.9$ & $4.5 \pm 0.6$ & 0.28 & $\begin{array}{c}-1.08(-3.03 / \\
0.88)\end{array}$ & 0.30 \\
\hline Enterotomy (\% $\pm S E)$ & $1.17 \pm 0.46$ & $0.97 \pm 0.28$ & 0.71 & $0.12(-0.93 / 1.17)$ & 0.82 \\
\hline GI bleed (\%土SE) & $0.39 \pm 0.27$ & $0.53 \pm 0.20$ & 0.69 & - & - \\
\hline Pulmonary insufficiency (\% $\%$ SE) & $0.78 \pm 0.38$ & $0.27 \pm 0.15$ & 0.13 & - & - \\
\hline Pulmonary failure $(\% \pm S E)$ & $1.6 \pm 0.5$ & $4.7 \pm 0.7$ & 0.002 & $\begin{array}{c}-3.80(-5.55 / \\
-2.04)\end{array}$ & $<0.0001$ \\
\hline Pneumonia (\% \pm SE) & $0.78 \pm 0.39$ & $0.97 \pm 0.29$ & 0.70 & $0.01(-1.06 / 1.07)$ & 0.99 \\
\hline Genitourinary complication (\% $\%$ SE) & $0.19 \pm 0.19$ & $0.27 \pm 0.15$ & 0.79 & - & - \\
\hline Postoperative infection (\% $\%$ SE) & $1.8 \pm 0.6$ & $2.5 \pm 0.5$ & 0.37 & $\begin{array}{c}-0.71(-2.48 / \\
1.07)\end{array}$ & 0.44 \\
\hline Wound complication (\% $\%$ SE) & $0.58 \pm 0.27$ & $0.35 \pm 0.18$ & 0.45 & $\begin{array}{c}-0.35(-1.84 / \\
1.14)\end{array}$ & 0.64 \\
\hline 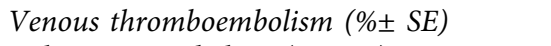 & $0.78 \pm 0.38$ & $0.44 \pm 0.20$ & 0.39 & - & - \\
\hline Pulmonary embolism (\% $\%$ SE) & $1.17 \pm 0.55$ & $0.35 \pm 0.17$ & 0.06 & $0.77(-0.47 / 2.00)$ & 0.22 \\
\hline "Other" complications $(\% \pm S E)$ & $0.19 \pm 0.19$ & $0.44 \pm 0.20$ & 0.44 & $\begin{array}{c}-0.39(-1.12 / \\
0.34)\end{array}$ & 0.29 \\
\hline Death $(\% \pm S E)$ & $0.58 \pm 0.34$ & $0.89 \pm 0.28$ & 0.52 & $\begin{array}{c}-4.91(-8.68 / \\
-1.15)\end{array}$ & 0.01 \\
\hline 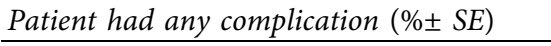 & $12.3 \pm 1.5$ & $16.4 \pm 1.1$ & 0.03 & - & - \\
\hline
\end{tabular}

HVBH: high volume bariatric hospital; LVBH: low volume bariatric hospital; ATE: average treatment effect.

failure $(3.22 \%$ vs. $0.49 \%, p=0.03)$ which persisted after propensity weighing $(p=0.02)$.

\section{Discussion}

This analysis of the NIS 2016 dataset shows a higher rate of several complications for patients with morbid obesity having general surgery operations at hospitals performing low volumes of bariatric surgery. The complication most consistently increased across the studied groups was pulmonary failure, with higher rates in patients undergoing elective laparoscopic cholecystectomy, elective ventral hernia repair, and appendectomy. Despite adjusting for general surgery hospital procedure volume and preoperative comorbidities, we found a significantly higher rate of mortality at LVBH after elective laparoscopic cholecystectomy and nonelective ventral hernia repair.

Overall, the rate of several complications in NIS for morbidly obese patients is higher than reported in the literature for the general population. Prior reports of the rate of 
TABLE 5: Preoperative comorbidities and postoperative complications of patients with morbid obesity undergoing nonelective ventral hernia repair.

\begin{tabular}{|c|c|c|c|c|c|}
\hline Preoperative variables & $\begin{array}{c}\text { HVBH } n=314 \\
\text { Weighted } \\
n=1570\end{array}$ & $\begin{array}{c}\text { LVBH } n=1143 \\
\text { Weighted } \\
n=5715\end{array}$ & $p$ value unadjusted & & \\
\hline Age (years, mean $\pm S E$ ) & $55.5 \pm 0.7$ & $56.4 \pm 0.4$ & 0.27 & & \\
\hline Length of stay (days, mean) & $8.0 \pm 0.6$ & $7.7 \pm 0.3$ & 0.56 & & \\
\hline Median household income quartile for zip code & $2.2 \pm 0.1$ & $2.2 \pm 0.0$ & 0.96 & & \\
\hline Gender, female (\%土SE) & $74.5 \pm 2.4$ & $70.1 \pm 1.3$ & 0.12 & & \\
\hline Renal failure (\% $\%$ SE) & $0.96 \pm 0.6$ & $1.4 \pm 0.4$ & 0.54 & & \\
\hline Hypertension (\%土SE) & $57.3 \pm 3.0$ & $58.4 \pm 1.4$ & 0.75 & & \\
\hline Chronic obstructive pulmonary dis. (\% $\$$ SE) & $17.8 \pm 2.2$ & $15.3 \pm 1.0$ & 0.29 & & \\
\hline Diabetes mellitus type $2(\% \pm S E)$ & $12.7 \pm 1.9$ & $11.7 \pm 0.9$ & 0.63 & & \\
\hline Anemia $(\% \pm S E)$ & $0.32 \pm 0.32$ & $0.79 \pm 0.26$ & 0.37 & & \\
\hline Gastroesophageal reflux disease (\% $\%$ SE) & $27.4 \pm 2.6$ & $22.9 \pm 1.2$ & 0.11 & & \\
\hline Chronic peptic ulcer disease (\% $\%$ SE) & $0.96 \pm 0.55$ & $0.61 \pm 0.23$ & 0.51 & & \\
\hline Heart failure (\% $\%$ SE) & $13.4 \pm 2.0$ & $12.7 \pm 1.0$ & 0.75 & & \\
\hline Hypothyroidism (\% $\%$ SE) & $14.6 \pm 2.0$ & $15.5 \pm 1.1$ & 0.72 & & \\
\hline History of venous thromboembolism (\% $\%$ SE) & $5.7 \pm 1.2$ & $4.8 \pm 0.6$ & 0.48 & & \\
\hline History of pulmonary embolism (\% $\$$ SE) & $5.7 \pm 1.4$ & $3.1 \pm 0.5$ & 0.03 & & \\
\hline Nicotine dependence $(\% \pm S E)$ & $21.7 \pm 2.5$ & $16.1 \pm 1.1$ & 0.03 & & \\
\hline Obstructive sleep apnea (\% $\%$ SE) & $35.7 \pm 2.7$ & $30.1 \pm 1.1$ & 0.06 & & \\
\hline Ventral hernia volume (cases/hospital $\pm S E$ ) & $20.9 \pm 1.8$ & $10.8 \pm 0.4$ & $<0.0001$ & & \\
\hline Postprocedure complications & $\mathrm{HVBH}$ & LVBH & $p$ value unadjusted & $\operatorname{ATE}(95 \% \mathrm{CI})$ & $\begin{array}{l}p \text { value } \\
\text { adjusted }\end{array}$ \\
\hline Shock $(\% \pm S E)$ & $0.64 \pm 0.32$ & $0.44 \pm 0.17$ & 0.56 & - & - \\
\hline Cardiac arrest $(\% \pm S E)$ & $0.32 \pm 0.32$ & $0.26 \pm 0.15$ & 0.87 & - & - \\
\hline Bowel obstruction (\%土SE) & $4.1 \pm 1.2$ & $5.5 \pm 0.7$ & 0.35 & $-0.79(-3.80 / 2.23)$ & 0.61 \\
\hline Enterotomy $(\% \pm S E)$ & $0.32 \pm 0.32$ & $0.79 \pm 0.26$ & 0.37 & $-0.45(-1.21 / 0.31)$ & 0.24 \\
\hline GI bleed $(\% \pm S E)$ & $0.96 \pm 0.55$ & $0.96 \pm 0.29$ & 0.99 & $-0.41(-1.33 / 0.52)$ & 0.39 \\
\hline Pulmonary insufficiency (\% $\$ S E)$ & $0.64 \pm 0.45$ & $0.26 \pm 0.15$ & 0.31 & - & - \\
\hline Pulmonary failure (\% $\mathrm{SE})$ & $4.1 \pm 1.1$ & $4.6 \pm 0.6$ & 0.70 & $-0.08(-2.81 / 2.66)$ & 0.96 \\
\hline Pneumonia (\% $($ SE) & $0.96 \pm 0.55$ & $0.61 \pm 0.23$ & 0.52 & $0.92(-0.83 / 2.67)$ & 0.30 \\
\hline Postoperative infection (\% $\%$ SE) & $2.6 \pm 0.9$ & $3.3 \pm 0.5$ & 0.47 & $-1.72(-3.49 / 0.06)$ & 0.06 \\
\hline Wound complication (\% $\%$ SE) & $0.32 \pm 0.32$ & $0.09 \pm 0.09$ & 0.33 & $-0.83(-2.94 / 1.27)$ & 0.44 \\
\hline Venous thromboembolism (\%土SE) & $1.59 \pm 0.70$ & $0.96 \pm 0.27$ & 0.33 & $0.15(-1.04 / 1.35)$ & 0.80 \\
\hline Pulmonary embolism $(\% \pm S E)$ & $1.9 \pm 0.8$ & $1.5 \pm 0.4$ & 0.59 & $-0.50(-1.74 / 0.75)$ & 0.43 \\
\hline Death $(\% \pm S E)$ & $0.32 \pm 0.32$ & $1.66 \pm 0.37$ & 0.07 & $\begin{array}{c}-1.15(-2.18 / \\
-0.12)\end{array}$ & 0.03 \\
\hline Patient had any complication (\% $\%$ SE) & $17.8 \pm 2.1$ & $17.8 \pm 1.1$ & 0.98 & $-2.49(-7.36 / 2.39)$ & 0.32 \\
\hline
\end{tabular}

HVBH: high volume bariatric hospital; LVBH: low volume bariatric hospital; ATE: average treatment effect.

mortality following laparoscopic cholecystectomy are between 0 and $0.13 \%$, compared to $0.23-0.96 \%$ in the NIS database [10]. Additionally, for laparoscopic cholecystectomy, the rate of any complication is reported as between $2.2 \%$ and $12 \%$ compared to 5.95 and $9.45 \%$ in patients with morbid obesity in the NIS database [10]. The rate of postoperative pulmonary failure in this current study $(2.53 \%-$ $4.53 \%)$ is similar to rates reported for patients undergoing bariatric surgery, with overall rates around $1.35 \%$ and the greatest rate of $4.1 \%$ after open gastric bypass [11]. This study highlights the overall increased complication rate for morbidly obese patients undergoing general surgery procedures.

The relationship between volumes and outcomes has been clearly observed but challenging at times to explain. Many studies have identified improved outcomes for patients undergoing surgery by surgeons who perform high volumes of complex surgery. Additionally, even when accounting for case mix, increased hospital case volume also was associated with improved outcomes independent of surgeon volume [12]. Specifically, for bariatric surgery, academic hospitals performing greater than 100 bariatric cases per year compared to those performing less than 50 per year had a shorter length of stay and fewer complications [7]. Bariatric surgery program site accreditation, which includes case-volume requirements, is associated with reduced morbidity and mortality [13,14]. Even among these accredited centers, the highest volume centers have the lowest rate of complications [15]. A systematic review of 24 papers on bariatric surgery volume and outcomes found both higher hospital volume and higher surgeon volume related to more positive outcomes [8]. The data in our study are unable to identify whether the surgeons performing the general surgery cases are also performing the bariatric surgeries or if it is other aspects of being a $\mathrm{HVBH}$ that improve general surgery outcomes [16]. 
TABLE 6: Preoperative comorbidities and postoperative complications of patients with morbid obesity undergoing nonelective appendectomy.

\begin{tabular}{|c|c|c|c|c|c|}
\hline Preoperative variables & $\begin{array}{c}\text { HVBH } n=205 \\
\text { Weighted } \\
n=1025\end{array}$ & $\begin{array}{c}\text { LVBH } n=870 \\
\text { Weighted } \\
n=4350\end{array}$ & $p$ value unadjusted & & \\
\hline Age (years, mean $\pm S E$ ) & $45.6 \pm 1.3$ & $44.4 \pm 0.6$ & 0.34 & & \\
\hline Length of stay (days, mean $\pm S E$ ) & $4.8 \pm 0.6$ & $3.9 \pm 0.1$ & 0.02 & & \\
\hline Median household income quartile for zip code & $2.2 \pm 0.1$ & $2.3 \pm 0.0$ & 0.05 & & \\
\hline Gender, female (\%土SE) & $40.5 \pm 3.5$ & $40.1 \pm 1.6$ & 0.92 & & \\
\hline Renal failure (\%土SE) & $1.5 \pm 0.8$ & $1.4 \pm 0.4$ & 0.93 & & \\
\hline Hypertension $(\% \pm S E)$ & $42.0 \pm 3.6$ & $41.0 \pm 1.6$ & 0.82 & & \\
\hline 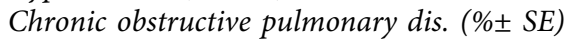 & $8.3 \pm 2.0$ & $7.2 \pm 0.9$ & 0.61 & & \\
\hline Diabetes mellitus type $2(\% \pm S E)$ & $9.8 \pm 2.1$ & $9.5 \pm 1.0$ & 0.92 & & \\
\hline Gastroesophageal reflux disease (\% $\%$ SE) & $18.0 \pm 2.7$ & $15.5 \pm 1.2$ & 0.37 & & \\
\hline Heart failure $(\% \pm S E)$ & $3.9 \pm 1.2$ & $5.8 \pm 0.8$ & 0.26 & & \\
\hline Hypothyroidism (\% SE) & $11.7 \pm 2.2$ & $8.7 \pm 0.9$ & 0.19 & & \\
\hline History of venous thromboembolism (\% $\%$ SE) & $1.5 \pm 0.8$ & $1.3 \pm 0.4$ & 0.82 & & \\
\hline History of pulmonary embolism (\% $\mathrm{SE}$ ) & $1.0 \pm 0.7$ & $1.2 \pm 0.4$ & 0.83 & & \\
\hline Nicotine dependence $(\% \pm S E)$ & $17.1 \pm 2.8$ & $13.2 \pm 1.1$ & 0.16 & & \\
\hline Obstructive sleep apnea (\% $\%$ SE) & $22.9 \pm 3.0$ & $19.7 \pm 1.4$ & 0.30 & & \\
\hline Appendectomy volume (cases/hospital $\pm S E$ ) & $23.1 \pm 1.4$ & $17.0 \pm 0.5$ & $<0.0001$ & & \\
\hline Postprocedure complications & $\mathrm{HVBH}$ & $\mathrm{LVBH}$ & $p$ value unadjusted & $\operatorname{ATE}(95 \% \mathrm{CI})$ & $\begin{array}{c}p \text { value } \\
\text { adjusted }\end{array}$ \\
\hline Bowel obstruction (\% $\%$ SE) & $3.4 \pm 1.3$ & $4.8 \pm 0.7$ & 0.38 & $-1.53(-4.60 / 1.55)$ & 0.33 \\
\hline Pulmonary insufficiency (\% $\%$ SE) & $0.49 \pm 0.49$ & $0.11 \pm 0.11$ & 0.26 & - & - \\
\hline Pulmonary failure $(\% \pm S E)$ & $0.49 \pm 0.49$ & $3.22 \pm 0.59$ & 0.03 & $\begin{array}{c}-2.28(-4.20 / \\
-0.35)\end{array}$ & 0.02 \\
\hline Pneumonia (\% $\$$ SE) & $0.49 \pm 0.48$ & $0.57 \pm 0.26$ & 0.88 & - & - \\
\hline 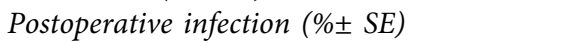 & $0.98 \pm 0.67$ & $0.69 \pm 0.28$ & 0.66 & - & - \\
\hline Venous thromboembolism (\% $\%$ SE) & $0.49 \pm 0.49$ & $0.23 \pm 0.16$ & 0.53 & - & - \\
\hline Pulmonary embolism (\% $\%$ SE) & $0.49 \pm 0.49$ & $0.46 \pm 0.23$ & 0.96 & - & - \\
\hline Death $(\% \pm S E)$ & $0.98 \pm 0.63$ & $0.23 \pm 0.16$ & 0.10 & - & - \\
\hline Patient had any complication $(\% \pm S E)$ & $6.8 \pm 2.0$ & $9.4 \pm 1.0$ & 0.28 & $-3.29(-7.23 / 0.64)$ & 0.10 \\
\hline
\end{tabular}

HVBH: high volume bariatric hospital; LVBH: low volume bariatric hospital; ATE: average treatment effect.

We used bariatric volume thresholds in the NIS as a surrogate for accreditation, as site-specific data is not available in the NIS. Our hypothesis was that HVBH, likely to be accredited, follow very specific standards and regulations to improve the quality of care for patients with morbid obesity. These pathways often extend beyond technical aspects of the operation, to include facilities that have ICU equipment, chairs, beds, doorways, and toilets that support bariatric weights. Staff are required to have sensitivity training, training on safe patient transfer and mobilization, and education about signs and symptoms of postoperative complications unique to these patients. Accredited bariatric centers are also required to participate in quality improvement projects and data registries [17]. A prior study using the NIS database during a time when hospitals were not deidentified found a threefold reduction in hospital mortality at accredited bariatric centers [18]. As our results suggest, a bariatric volume to general surgery outcome relationship, it may be that the multimodality pathways required for accreditation at high volume bariatric centers create a culture of improved safety and outcomes for many general surgery procedures performed in morbidly obese patients.

The greatest source of increased morbidity for patients at LVBH was postprocedure respiratory failure. Risk factors for postprocedure respiratory failure include peripheral vascular disease, age $>50$, alcohol use, diabetes mellitus, smoking, obstructive sleep apnea, and chronic lung disease [11]. Patients with morbid obesity can have functional respiratory changes from surplus adipose tissue that causes reduced functional residual capacity and expiratory reserve volume [19]. In patients with morbid obesity, the nonhypoxic apnea time is reduced from three minutes in normal weight patients to one minute, and in the supine position, expiratory lung volume is reduced by $69 \%$ after the induction of anesthesia [20, 21]. Using a ramped or head elevated position for patients with morbid obesity has been shown to improve pulmonary compliance, allow for easier mask ventilation, and improve conditions for tracheal intubation by lengthening the time to desaturation by 50 seconds and increasing the likelihood of a grade one view by $30 \%[22,23]$. Additionally, patients are less likely to receive intraoperative lung protective ventilation with high volumes and low positive end-expiratory pressure [24]. This has been associated with higher rates of postprocedure pulmonary complications including unplanned need for oxygen (if not part of usual patient care), unexpected postoperative invasive or noninvasive mechanical ventilation, acute respiratory failure, acute respiratory distress syndrome, pneumonia, and pneumothorax. While the best ventilatory strategy for 
patients with morbid obesity is unknown, likely, anesthesia staff who are experienced with the management of morbidly obese patients result in a volume-outcome relationship for improved postoperative pulmonary outcomes [25].

The main limitations of this study are due to the nature of NIS collection. The NIS data is collected for analysis of costs and charges related to healthcare, not for quality purposes. Complication rates in NIS are higher than complications rates in NSQIP, attributed to the difference in data entry. For this study, it was necessary to use the NIS data because NSQIP and other databases do not associate the patients with a specific hospital, a feature of the NIS data that was crucial to perform this analysis. The ICD-10 codes are coded at hospital discharge and different states submit different code numbers to the NIS for collection in the data set. If a state only submits 10 codes, then both comorbid conditions and postoperative complications may be missed; however, this omission should be similar between the investigated cohorts. While it is widely believed that there is underreporting of obesity and other medical comorbidities in these databases, it is not clear which way would influence these results or if the large number of hospitals in each group would balance this confounding. Additionally, many cases of laparoscopic cholecystectomy, appendectomy, and ventral hernia repair may be conducted as outpatient surgery and are not included in this analysis. This means that included patients likely represent a sicker cohort than the overall cohort of patients seen at each set of hospitals. And lastly, a significant limitation of the NIS is that NIS approximates a 20 -percent stratified sample of discharges from hospitals rather than a true census of discharges, so the categorization of hospital volume could be flawed and should and will be verified in future studies by databases that can provide a precise census of discharges from individual hospitals, such as individual state data through the HCUP Central Distributor. While these limitations are significant, we believe the outcome differences seen between the two groups are robust enough that the importance should not be dismissed. These descriptive results are compelling that there is room for improvement in the care of the general surgery patient with morbid obesity.

\section{Conclusions}

Patients with morbid obesity may have an advantage in having general surgery procedures performed at $\mathrm{HVBH}$. HVBH may have a volume-outcomes relationship where the hospital and staff familiarity with the management principles required to minimize the postoperative risk associated with morbid obesity improve patient outcomes for general surgery procedures.

\section{Data Availability}

The data are available from the Healthcare Cost and Utilization Project: https://www.hcup-us.ahrq.gov/db/nation/ nis/nisdbdocumentation.jsp.

\section{Ethical Approval}

This study was exempted from ethics review based on the Institutional Review Board policy on database research involving the National Inpatient Sample data.

\section{Conflicts of Interest}

The authors declare that they have no conflicts of interest.

\section{Authors' Contributions}

KHW, TK, and JG devised the project, the main conceptual ideas, and proposed methods. RW and AS performed the statistical analysis and create the propensity model. All the authors discussed the results and contributed to the final manuscript.

\section{Acknowledgments}

The authors would like to thank Dr. Rachel Budithi for her insight and feedback on anesthesia management of the morbidly obese patient. There are no funding sources to disclose.

\section{Supplementary Materials}

Table A: ICD 10 codes for postoperative complications. (Supplementary Materials)

\section{References}

[1] A. Sood, F. Abdollah, J. D. Sammon et al., "The effect of body mass index on perioperative outcomes after major surgery: results from the national surgical quality improvement program (ACS-NSQIP) 2005-2011," World Journal of Surgery, vol. 39, no. 10, pp. 2376-2385, 2015.

[2] P. Ferrada, R. J. Anand, A. Malhotra, and M. Aboutanos, "Obesity does not increase mortality after emergency surgery," Journal of Obesity, vol. 2014, Article ID 492127, 3 pages, 2014.

[3] C. Stroh, N. Michel, N. Michel et al., "Risk of thrombosis and thromboembolic prophylaxis in obesity surgery: data analysis from the German bariatric surgery registry," Obesity Surgery, vol. 26, no. 11, pp. 2562-2571, 2016.

[4] H.-T. Lin, P.-C. Ting, W.-Y. Chang, M.-W. Yang, C.-J. Chang, and A.-H. Chou, "Predictive risk index and prognosis of postoperative reintubation after planned extubation during general anesthesia: a single-center retrospective case-controlled study in Taiwan from 2005 to 2009," Acta Anaesthesiologica Taiwanica, vol. 51, no. 1, pp. 3-9, 2013.

[5] T. M. Cook, N. Woodall, and C. Frerk, "Major complications of airway management in the UK: results of the fourth national audit project of the royal college of anaesthetists and the difficult airway society. part 1: anaesthesia," British Journal of Anaesthesia, vol. 106, no. 5, pp. 617-631, 2011.

[6] L. Owei, R. A. Swendiman, R. R. Kelz, D. T. Dempsey, and K. R. Dumon, "Impact of body mass index on open ventral hernia repair: a retrospective review," Surgery, vol. 162, no. 6, pp. 1320-1329, 2017.

[7] N. T. Nguyen, M. Paya, C. M. Stevens, S. Mavandadi, K. Zainabadi, and S. E. Wilson, "The relationship between hospital volume and outcome in bariatric surgery at academic 
medical centers," Annals of Surgery, vol. 240, no. 4, pp. 586-594, 2004.

[8] B. Zevin, R. Aggarwal, and T. P. Grantcharov, "Volumeoutcome association in bariatric surgery: a systematic review," Annals of Surgery, vol. 256, no. 1, pp. 60-71, 2012.

[9] G. Banka, G. Woodard, T. Hernandez-Boussard, and J. M. Morton, "Laparoscopic vs open gastric bypass surgery: differences in patient demographics, safety, and outcomes," Archives of Surgery, vol. 147, no. 6, pp. 550-556, 2012.

[10] V. S. Lee, R. S. Chari, G. Cucchiaro, and W. C. Meyers, "Complications of laparoscopic cholecystectomy," The American Journal of Surgery, vol. 165, no. 4, pp. 527-532, 1993.

[11] H. Masoomi, K. M. Reavis, B. R. Smith, H. Kim, M. J. Stamos, and N. T. Nguyen, "Risk factors for acute respiratory failure in bariatric surgery: data from the nationwide inpatient sample, 2006-2008," Surgery for Obesity and Related Diseases, vol. 9, no. 2, pp. 277-281, 2013.

[12] J. W. Harmon, D. G. Tang, T. A. Gordon et al., "Hospital volume can serve as a surrogate for surgeon volume for achieving excellent outcomes in colorectal resection," Annals of Surgery, vol. 230, no. 3, pp. 404-411, 1999.

[13] J. M. Morton, T. Garg, and N. Nguyen, "Does hospital accreditation impact bariatric surgery safety?" Annals of Surgery, vol. 260, no. 3, pp. 504-509, 2014.

[14] D. Azagury and J. M. Morton, "Bariatric surgery outcomes in US accredited vs non-accredited centers: a systematic review," Journal of the American College of Surgeons, vol. 223, no. 3, pp. 469-477, 2016.

[15] A. M. Ibrahim, A. A. Ghaferi, J. R. Thumma, and J. B. Dimick, "Variation in outcomes at bariatric surgery centers of excellence," JAMA Surgery, vol. 152, no. 7, pp. 629-636, 2017.

[16] A. Pakula and R. Skinner, "Do acute care surgeons need bariatric surgical training to ensure optimal outcomes in obese patients with nonbariatric emergencies?" Surgery for Obesity and Related Diseases, vol. 14, no. 3, pp. 339-341, 2018.

[17] American College of Surgeons, Optimal Resources for Metabolic and Bariatric Surgery, American College of Surgeons, Chicago, IL, USA, 2019.

[18] A. Gebhart, M. Young, M. Phelan, and N. T. Nguyen, "Impact of accreditation in bariatric surgery," Surgery for Obesity and Related Diseases, vol. 10, no. 5, pp. 767-773, 2014.

[19] J. L. Pépin, J. F. Timsit, R. Tamisier, J. C. Borel, P. Lévy, and S. Jaber, "Prevention and care of respiratory failure in obese patients," The Lancet Respiratory Medicine, vol. 4, no. 5, pp. 407-418, 2016.

[20] A. De Jong, E. Futier, A. Millot et al., "How to preoxygenate in operative room: healthy subjects and situations "at risk"," Annales Françaises d'Anesthesie et de Reanimation, vol. 33, no. 7-8, pp. 457-461, 2014.

[21] E. Futier, J.-M. Constantin, A. Petit et al., "Positive end-expiratory pressure improves end-expiratory lung volume but not oxygenation after induction of anaesthesia," European Journal of Anaesthesiology, vol. 27, no. 6, pp. 508-513, 2010.

[22] B. J. Dixon, J. B. Dixon, J. R. Carden et al., "Preoxygenation is more effective in the 25 degrees head-up position than in the supine position in severely obese patients: a randomized controlled study," Anesthesiology, vol. 102, no. 6, pp. 1110-1115, 2005.

[23] J. S. Collins, H. J. M. Lemmens, J. B. Brodsky, J. G. Brock-utne, and R. M. Levitan, "Laryngoscopy and morbid obesity: a comparison of the "sniff" and "ramped" positions," Obesity Surgery, vol. 14, no. 9, pp. 1171-1175, 2004.

[24] L. Ball, S. N. T. Hemmes, A. Serpa Neto et al., "Intraoperative ventilation settings and their associations with postoperative pulmonary complications in obese patients," British Journal of Anaesthesia, vol. 121, no. 4, pp. 899-908, 2018.

[25] U. Shah, J. Wong, D. T. Wong, and F. Chung, "Preoxygenation and intraoperative ventilation strategies in obese patients: a comprehensive review," Current Opinion in Anaesthesiology, vol. 29, no. 1, pp. 109-118, 2016. 